\title{
Efficacy and safety of vildagliptin,
} sitagliptin, and linagliptin as add-on therapy in Chinese patients with T2DM inadequately controlled with dual combination of insulin and traditional oral hypoglycemic agent

Yun-Zhao Tang, Gang Wang, Zhen-Huan Jiang, Tian-Tian Yan, Yi-Jun Chen, Min Yang, Ling-Ling Meng, Yan-Juan Zhu, Chen-Guang Li, Zhu Li, Ping Yu and Chang-Lin Ni*

\begin{abstract}
Objective: We aimed to evaluate the efficacy and safety of the three dipeptidyl peptidase 4 (DPP-4) inhibitors (vildagliptin, sitagliptin, and linagliptin) as add-on therapy in Chinese patients with type 2 diabetes mellitus (T2DM) inadequately controlled on dual combination of insulin and metformin or acarbose.

Methods: A total of 535 T2DM patients who failed to achieve glycemic control with insulin and a traditional oral hypoglycemic agent were randomized to receive vildagliptin, sitagliptin, or linagliptin. Body mass index, glycosylated hemoglobin ( $\mathrm{HbA} 1 \mathrm{c})$, fasting and postprandial plasma glucose (FPG and PPG), insulin dose, and adverse events were evaluated during the study.

Results: The baseline HbA1c was $9.59 \pm 1.84 \%$ (vildagliptin group), $9.22 \pm 1.60 \%$ (sitagliptin group), and $9.58 \pm 1.80 \%$ (linagliptin group). At week 12 it was $8.16 \pm 1.29 \%$ (vildagliptin), $8.56 \pm 1.96 \%$ (linagliptin), and $8.26 \pm 1.10 \%$ (sitagliptin). The changes in HbA1c from baseline were $-1.33 \pm 0.11 \%$ (vildagliptin), $-0.84 \pm 0.08 \%$ (sitagliptin) and $-0.81 \pm 0.08 \%$ (linagliptin), the vildagliptin group had the greatest reduction in $\mathrm{HbA} 1 \mathrm{c}(P<0.05)$. The proportions of patients that reached target HbA1c were $66.27 \%$ (vildagliptin), $52.73 \%$ (sitagliptin), and $55.49 \%$ (linagliptin), the vildagliptin group had the highest one $(P<0.05)$. The baseline FPG and PPG values in the three groups were at the same level. At week 12, mean FPG levels in the vildagliptin ( $7.31 \pm 1.50 \mathrm{mmol} / \mathrm{L})$ and linagliptin $(6.90 \pm 1.55 \mathrm{mmol} / \mathrm{L})$ groups were significantly lower than in the sitagliptin group $(8.02 \pm 4.48 \mathrm{mmol} / \mathrm{L} ; P<0.05)$; the linagliptin group had the lowest mean PPG followed by the vildagliptin group which was also significant lower $(P=0.000)$ than the sitagliptin group. Additionally, the required insulin dosage in the vildagliptin group was the lowest among the groups at weeks 6 and 12 . Only mild AEs were reported during the study.
\end{abstract}

Conclusion: The three DPP-4 inhibitors appear to be effective and safe as add-on therapy for T2DM patients on dual combination of insulin and a traditional OHA. Vildagliptin was more effective in decreasing insulin requirement and achieving glycemic control when compared to the other two.

Keywords: Add-on therapy to insulin, Type 2 diabetes mellitus, DPP-4 inhibitors, Glycemic control

\footnotetext{
*Correspondence: ncl1959@163.com

Key Laboratory of Hormones and Development (Ministry of Health),

Metabolic Diseases Hospital and Tianjin Institute of Endocrinology,

Tianjin Medical University, Tongan Road 66, Heping District,

Tianjin 300070, China
} 


\section{Background}

Type 2 diabetes mellitus (T2DM) affects over 300 million people worldwide [1]. The global prevalence of T2DM was estimated to be $9 \%$ among adults aged over 18 years in 2014 [1]. Excluding accidents, diabetes is the fifth cause of death for women and the fourth for men in the USA [2]. In China, the total diabetes prevalence was $9.7 \%$ (92.4 million adults) according to the China National Diabetes and Metabolic Disorders Study between 2007 and 2008, while the prevalence of prediabetes was estimated to be $15.5 \%$ (148 million adults) [3]. The increasing prevalence of diabetes has followed rapid economic growth, increases in life expectancy, and changes in lifestyle [3]. Inadequate control of blood glucose in patients correlates with a higher risk for diabetes-related micro and macrovascular complications $[4,5]$. The management of diabetes aims at improving glycemic control to reduce the onset of complications [6]. Glycemic control is typically measured as reductions in glycosylated hemoglobin (HbA1c).

T2DM is a progressive disease that often requires a combination of antidiabetic drugs with different mechanisms of action to achieve glycemic targets over time [7]. Dipeptidyl peptidase 4 (DPP-4) inhibitors have become a useful class of oral hypoglycemic agents (OHA) for the treatment of T2DM since 2006. DPP-4 is a transmembrane glycoprotein located on the surface of most cell types, and its multiple effects may be associated with immune regulation, cell apoptosis, and signal transduction [8]. The clinically relevant action of DPP-4 is the degradation of endogenous glucagon-like peptide 1 (GLP-1). Additionally, DPP-4 inhibitors enhance insulin secretion in a glucose-dependent manner [9].

Several clinical practice guidelines recommend a stepwise treatment pathway for diabetes. Diet control and lifestyle intervention are considered the cornerstones for treatment of DM according to these guidelines. However, dietary and lifestyle changes are difficult to implement and maintain on a large scale. Given the progressive nature of T2DM, long-term glycemic control is difficult to achieve with a single agent, thus often requiring the addition of further agents [10]. The American Diabetes Association/European Association for the Study of Diabetes position statement and the American Association of Clinical Endocrinologists/American College of Endocrinology algorithm suggest the use of DPP-4 inhibitors as a second option when metformin fails [11]. Addition of a DPP-4 enzyme inhibitor to metformin treatment is beneficial owing to the complementary mechanisms of action of both drugs [11-14]. Nonetheless, the role of those new drugs in the treatment of T2DM is still debated [15]. A few clinical studies have reported the effect of DPP-4 inhibitors as an add-on therapy to insulin
[16-18]. The efficacy in achieving glycemic control and weight-sparing effects of DPP-4 inhibitors have been stated in both systematic reviews and meta-analyses in comparison with placebo and other antidiabetic medications [19-21]. However, their safety and the efficacy when combined with traditional anti-hyperglycemic therapy remain inconclusive. Therefore, we conducted this study to evaluate the efficacy and safety of DPP-4 inhibitor vildagliptin, sitagliptin, or linagliptin as add-on therapy for T2DM patients inadequately controlled with dual combination of insulin and metformin or acarbose.

\section{Methods}

\section{Subjects}

Patients were recruited from the Metabolic Diseases Hospital of Tianjin Medical University between January 2013 and January 2015. Enrolled male or female patients met the following criteria: diagnosis of T2DM; age >18 years; HbA1c levels $>7.0 \%$; body mass index (BMI) between 22 and $45 \mathrm{~kg} / \mathrm{m}^{2}$ at visit 1 (week -4); current treatment with insulin at a stable dose of 20-80 U daily and a traditional OHA (metformin 750-1000 mg daily or acarbose 100$300 \mathrm{mg}$ daily) for at least 12 weeks before screening.

Patients who met the following criteria were excluded from the study: pregnant or lactating women; diagnosis of type 1 diabetes mellitus, diabetes secondary to pancreatic injury or other types of secondary diabetes; acute metabolic diabetic complications, such as ketoacidosis or hyperosmolar state (coma) within the 3 months prior to enrollment; myocardial infarction, unstable angina or coronary artery bypass surgery within 6 months prior to enrollment; congestive heart failure (NYHA III-IV grade); history of liver disease such as cirrhosis, hepatitis $\mathrm{B}$, or hepatitis $\mathrm{C}$ (except carriers), or alanine transaminase (ALT), aspartate aminotransferase (AST) greater than two times the upper limit of normal (ULN) or total bilirubin greater than two times the ULN; history of kidney disease or clinical diagnosis of renal insufficiency indicated by serum creatinine $\geq 132 \mu \mathrm{mol} / \mathrm{L}(\geq 1.5 \mathrm{mg} /$ $\mathrm{dL})$ in male patients, and $\geq 123 \mu \mathrm{mol} / \mathrm{L}(\geq 1.4 \mathrm{mg} / \mathrm{dL})$ in female patients; thyroid-stimulating hormone beyond the normal range; and history of acute and chronic pancreatitis.

Written informed consent was obtained from each patient prior to enrollment. This study design was approved by the local ethics committee and review board and was in accordance with the Declaration of Helsinki.

\section{Study design}

This was a 12-week, randomized, open-label, parallel clinical study. The flow diagram of the study design is presented in Fig. 1. Patients with T2DM who met the inclusion criteria were screened for eligibility 


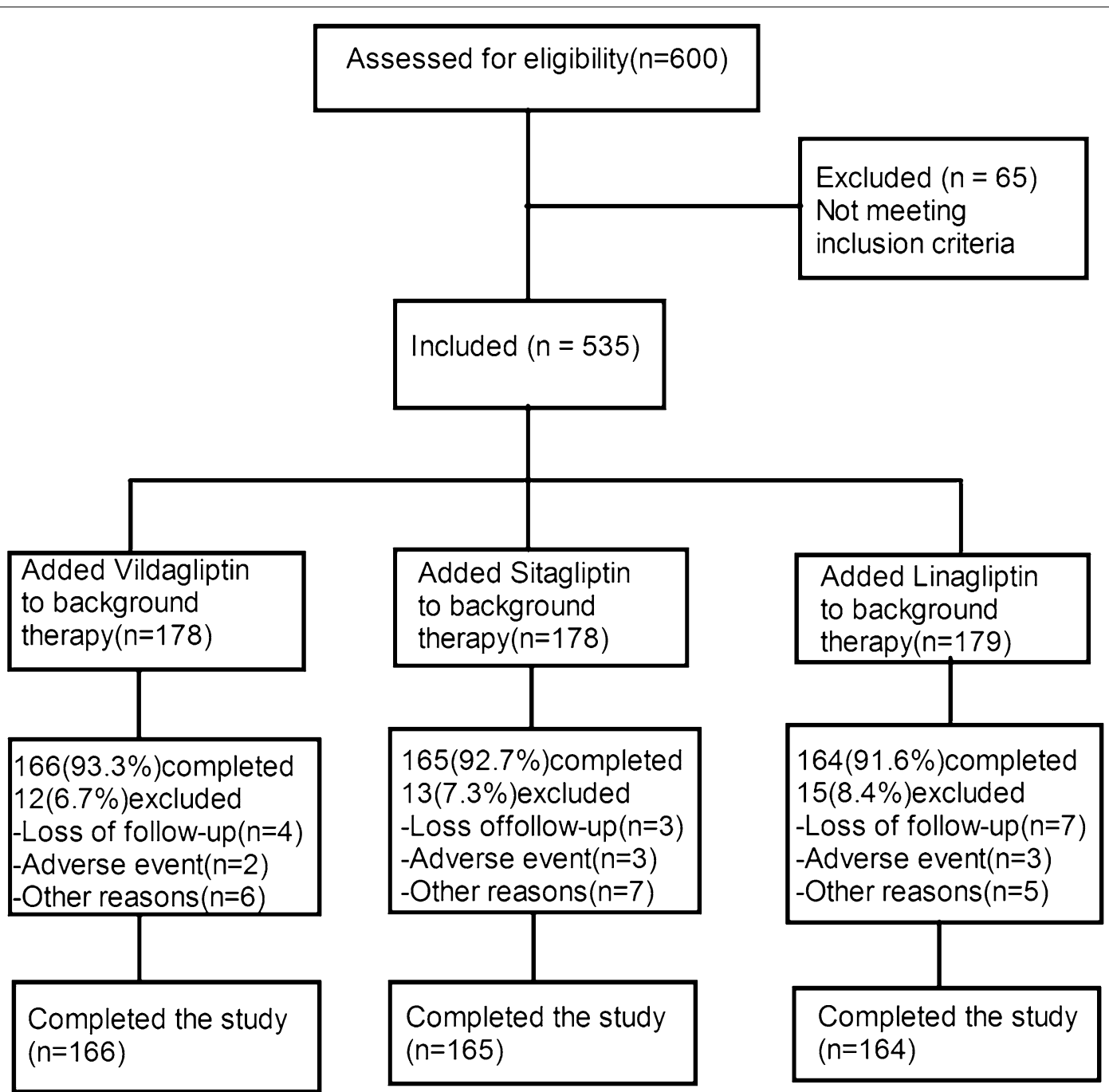

Fig. 1 Flow diagram of the patient recruitment process

at Visit 1 (week -4) and were randomized (1:1:1) at Visit 2 (week 0 , baseline) to receive vildagliptin $50 \mathrm{mg}$ bid, sitagliptin $100 \mathrm{mg}$ qd, or linagliptin $5 \mathrm{mg}$ qd for 12 weeks. Patients were required to maintain their individual eating and exercise habits during the study, and follow all the study requirements as well. At and after the screening visit, baseline laboratory and clinical data were evaluated in every case. The DPP-4 inhibitor was administered as an add-on therapy to the background OHA maintained throughout the study. The patients were followed up every 2 weeks in the outpatient department. Dose adjustments of insulin or analogs were performed by the treating physicians according to the level of the blood glucose at each visit.

\section{Study assessments and endpoints}

The primary study endpoint was change in HbA1c and the proportion of patients that reached the target $\mathrm{HbA1c}$ level (<7.0 \%) [22] from baseline to week 12. Secondary efficacy assessments included changes in FPG, PPG, and the insulin dose from baseline to endpoint (week 12). Safety assessments included recording and monitoring of treatment-emergent adverse events (AEs); biochemistry and hematology laboratory test results; electrocardiogram findings and vital signs. Hypoglycemia was defined as symptoms suggestive of hypoglycemia with a selfmonitored plasma glucose measurement $<3.1 \mathrm{mmol} / \mathrm{L}$. Severe hypoglycemia was defined as an episode requiring external assistance or hospitalization with or without a plasma glucose measurement $<3.1 \mathrm{mmol} / \mathrm{L}$. 


\section{Statistical analysis}

All the measurement data were expressed as mean \pm standard deviation. One-way analysis of variance was used to compare the differences in clinical characteristics between the three groups at baseline and after treatment. Fisher's least significant difference test was adopted for multiple comparisons. Chi square test was performed for analysis of differences in the frequency distributions. The data were analyzed by SPSS 18.0 for Windows (SPSS Inc., Chicago, IL, USA). $P<0.05$ was considered statistically significant.

\section{Results}

\section{Patient Disposition and Baseline Characteristics}

The disposition of patients from screening to study endpoint is depicted in Fig. 1. Of total 600 patients screened, 535 were randomized to receive vildagliptin $(n=178)$, sitagliptin $(\mathrm{n}=178)$, and linagliptin $(\mathrm{n}=179)$ respectively. 166 of $178(93.3 \%)$ patients in the vildagliptin group, 165 of 178 (92.7\%) in the sitagliptin group, and 164 of 179 (91.6\%) in the linagliptin group completed the study. Discontinuation in the three groups was mainly due to loss to follow-up, adverse events (mainly hypoglycemia), or other reasons.

The baseline characteristics of the randomized patients are presented in Table 1 . The three groups were well balanced at baseline in terms of gender, age, BMI, disease duration, insulin dose and background therapy (insulin and OHA). All biochemistry indexes were matched between groups except for blood urea nitrogen (BUN).

\section{Efficacy}

At baseline HbA1c was $9.59 \pm 1.84 \%$ in the vildagliptin group, $9.22 \pm 1.60 \%$ in the sitagliptin group, and $9.58 \pm 1.80 \%$ in the linagliptin group, no differences was found between the three groups $(P=0.097)$. After 12 weeks of treatment, it was reduced to $8.16 \pm 1.29 \%$ (vildagliptin), $8.56 \pm 1.96 \%$ (linagliptin), and $8.26 \pm 1.10 \%$ (sitagliptin). Except that the vildagliptin group had a lower HbA1c value than the linagliptin group $(P=0.044)$, no significant differences in HbA1c were found between the groups (Fig. 2a).

The change in HbA1c from baseline was the most important end point of our study. As mentioned above all three groups had a decline in $\mathrm{HbA1c}$ but the vildagliptin group had the greatest one $(-1.33 \pm 0.11 \%)$ $(P=0.000)$. The changes in HbA1c were $-0.84 \pm 0.08$ and $-0.81 \pm 0.08 \%$ in the sitagliptin and linagliptin groups, respectively (Fig. 2b).

$66.27 \%$ of patients in the vildagliptin group achieved target HbA1c level, whereas in sitagliptin group the proportion was $52.73 \%$ and in the linagliptin group $55.49 \%$. The vildagliptin group had the highest proportion that reached target HbA1c among the three groups $(\mathrm{P}<0.05)$ (Fig. 2c).

Mean FPG values during the 12 weeks of treatment are presented in Fig. 3a. The baseline values in the three groups were at the same level. At week 6 , they were $6.68 \pm 1.03 \mathrm{mmol} / \mathrm{L}$ in the vildagliptin group, $7.22 \pm 1.47 \mathrm{mmol} / \mathrm{L}$ in the sitagliptin group, and $6.95 \pm 1.27 \mathrm{mmol} / \mathrm{L}$ in the linagliptin group. All three groups revealed a decline in FPG when compared with their baseline levels, but it was more significant in the vildagliptin arm than in the other two groups $(\mathrm{P}=0.001)$. At week 12, mean FPG levels were $7.31 \pm 1.50 \mathrm{mmol} / \mathrm{L}$ in the vildagliptin group and $6.90 \pm 1.55 \mathrm{mmol} / \mathrm{L}$ in the linagliptin group, significantly lower when compared with the sitagliptin group $(8.02 \pm 4.48 \mathrm{mmol} / \mathrm{L} ; \mathrm{P}=0.002)$.

The mean PPG values during the 12 weeks of the study are depicted in Fig. 3b. The values at baseline were $15.05 \pm 4.02 \mathrm{mmol} / \mathrm{L}$ in the vildagliptin group, $14.82 \pm 3.58 \mathrm{mmol} / \mathrm{L}$ in the sitagliptin group, and $15.21 \pm 3.78 \mathrm{mmol} / \mathrm{L}$ in the linagliptin group. No differences were found between the three groups $(P=0.653)$. All three groups revealed a reduction in PPG after treatment, but to a slightly different degree. At week 6, the mean PPG was $9.09 \pm 1.83 \mathrm{mmol} / \mathrm{L}$ (vildagliptin), $9.16 \pm 2.24 \mathrm{mmol} / \mathrm{L}$ (linagliptin) and $9.80 \pm 2.23 \mathrm{mmol} / \mathrm{L}$ (sitagliptin). The value was significantly lower in the vildagliptin group and linagliptin group than in the sitagliptin group $(P=0.004)$. At week 12 , the value was $9.03 \pm 2.53 \mathrm{mmol} / \mathrm{L}$ (linagliptin), $9.80 \pm 2.22 \mathrm{mmol} / \mathrm{L}$ (vildagliptin) and $10.58 \pm 2.64 \mathrm{mmol} / \mathrm{L}$ (sitagliptin). The linagliptin group had the lowest mean PPG followed by the vildagliptin group. Although the value in the vildagliptin group was not as low as in the linagliptin group, it was significantly lower $(P=0.000)$ than that in the sitagliptin group.

A decline in mean insulin dose was noticed in the three groups over the 12 -week study period. At baseline, the dose was $33.15 \pm 12.89 \mathrm{U}$ in the vildagliptin group, $36.02 \pm 13.09 \mathrm{U}$ in the sitagliptin group, and $33.43 \pm 15.76 \mathrm{U}$ in the linagliptin group $(\mathrm{P}=0.121)$. At week 6 the dose was $23.92 \pm 0.96 \mathrm{U}$ (vildagliptin), $24.89 \pm 1.28 \mathrm{U}$ (linagliptin) and $28.29 \pm 1.06 \mathrm{U}$ (sitagliptin). The vildagliptin group had the lowest insulin dose. The dose in the linagliptin group, though not as low as in the vildagliptin group, was lower than in the sitagliptin group $(\mathrm{P}=0.014)$. At week 12 the dose was $20.71 \pm 12.36 \mathrm{U}$ (vildagliptin), 27.34 $\pm 13.46 \mathrm{U}$ (sitagliptin) and $24.81 \pm 15.08 \mathrm{U}$ (linagliptin). Comparison between groups showed that the mean insulin dose was much lower in the vildagliptin group than in the other two groups $(P=0.000)$ (Fig. 4a).

In the present study change in insulin dose was an important variable which demonstrated a downward 
Table 1 Baseline characteristics of the participants

\begin{tabular}{|c|c|c|c|c|c|}
\hline Variables & Vildagliptin ( $\mathrm{n}=166$ ) & Sitagliptin $(n=165)$ & Linagliptin $(n=164)$ & For $\mathrm{x}^{2}$ values & $P$ values \\
\hline Male, n (\%) & $92(55.4)$ & $104(63.0)$ & $104(63.4)$ & 2.817 & 0.245 \\
\hline Age (years) & $56.20 \pm 10.37$ & $56.04 \pm 13.80$ & $54.66 \pm 10.85$ & 0.851 & 0.428 \\
\hline $\mathrm{BMI}\left(\mathrm{kg} / \mathrm{cm}^{2}\right)$ & $26.49 \pm 6.44$ & $26.51 \pm 4.29$ & $26.82 \pm 4.80$ & 0.198 & 0.821 \\
\hline Disease duration (years) & $7.78 \pm 0.58$ & $8.50 \pm 0.62$ & $7.51 \pm 0.53$ & 0.779 & 0.459 \\
\hline $\mathrm{SBP}(\mathrm{mmHg})$ & $131.63 \pm 16.41$ & $130.92 \pm 15.32$ & $131.53 \pm 18.71$ & 0.085 & 0.918 \\
\hline $\mathrm{DBP}(\mathrm{mmHg})$ & $79.85 \pm 8.92$ & $80.33 \pm 7.93$ & $81.38 \pm 11.02$ & 1.122 & 0.326 \\
\hline $\mathrm{ALT}(\mathrm{IU} / \mathrm{L})$ & $23.98 \pm 13.83$ & $23.87 \pm 14.56$ & $27.34 \pm 16.70$ & 2.397 & 0.092 \\
\hline AST (IU/L) & $20.51 \pm 9.36$ & $22.75 \pm 10.64$ & $22.01 \pm 10.98$ & 1.898 & 0.151 \\
\hline BUN (mmol/L) & $5.93 \pm 1.79$ & $6.32 \pm 2.58$ & $5.49 \pm 1.35$ & 5.639 & 0.004 \\
\hline $\mathrm{Cr}(\mathrm{umol} / \mathrm{L})$ & $66.08 \pm 45.22$ & $69.09 \pm 23.88$ & $64.08 \pm 13.88$ & 0.880 & 0.416 \\
\hline $\mathrm{TG}(\mathrm{mmol} / \mathrm{L})$ & $2.14 \pm 2.01$ & $2.29 \pm 1.82$ & $2.26 \pm 1.72$ & 0.286 & 0.751 \\
\hline $\mathrm{TC}(\mathrm{mmol} / \mathrm{L})$ & $5.16 \pm 1.24$ & $5.30 \pm 1.36$ & $5.09 \pm 1.04$ & 1.017 & 0.363 \\
\hline $\mathrm{HbA1c}(\%)$ & $9.59 \pm 1.84$ & $9.22 \pm 1.60$ & $9.58 \pm 1.80$ & 2.345 & 0.097 \\
\hline Insulin dose $(U)$ & $33.15 \pm 1.00$ & $36.02 \pm 1.06$ & $33.43 \pm 1.23$ & 2.121 & 0.121 \\
\hline \multicolumn{6}{|l|}{ Insulin therapy, n (\%) } \\
\hline Premixed human insulin & $65(39.15)$ & $81(49.00)$ & $64(39.02)$ & 7.270 & 0.122 \\
\hline Premixed insulin analogs & $42(25.30)$ & $44(26.67)$ & $41(25.00)$ & & \\
\hline Basal insulin & $59(35.54)$ & $40(25.32)$ & $59(35.98)$ & & \\
\hline \multicolumn{6}{|l|}{ Background OHA, n (\%) } \\
\hline Metformin & $80(48.2)$ & $93(56.4)$ & $90(54.9)$ & 2.519 & 0.284 \\
\hline Acarbose & $86(51.8)$ & $72(43.6)$ & $74(45.1)$ & & \\
\hline
\end{tabular}

Values are expressed as mean \pm SD or $\mathrm{n}(\%)$. BUN in the linagliptin group was lower than the other two groups $(P<0.05)$

$A L T$ alanine transaminase, $A S T$ aspartate transaminase, $B M I$ body mass index, BUN blood urea nitrogen, $S B P$ systolic blood pressure, $C r$ creatinine, $D B P$ diastolic blood pressure, $\mathrm{OHA}$ oral hypoglycemic agent, $T C$ total cholesterol, $T G$ triglyceride

trend in the three groups. At week 6, the change was $-9.17 \pm 0.95 \mathrm{U}$ in the vildagliptin group, $-7.73 \pm 0.86$ $\mathrm{U}$ in the sitagliptin group, and $-8.85 \pm 0.88 \mathrm{U}$ in the linagliptin group. No differences were found between the three groups. At week 12, however, the change was $-12.24 \pm 1.11 \mathrm{U}$ (vildagliptin), $-12.81 \pm 1.13 \mathrm{U}$ (sitagliptin) and $-8.63 \pm 0.93 \mathrm{U}$ (linagliptin). The change was more pronounced in the vildagliptin group and sitagliptin group than in the linagliptin group $(\mathrm{P}=0.013)$ (Fig. 4b).

We measured the participants' BMI, blood pressure, and lipid profile during the 12-week follow-up. There were no changes in BMI and blood pressure. The total cholesterol (TC) and triglycerides (TG) in the three groups showed a downtrend when compared with the baseline, but the differences were not significant. In addition no differences in TC and TG at week 6 or 12 were found between the groups (data not shown).

\section{Safety}

No severe AEs were reported in the three groups. All the AEs reported during the study were mild. The most commonly reported AEs were gastrointestinal AEs (14.46\% for vildagliptin, $11.52 \%$ for sitagliptin, and $9.15 \%$ for linagliptin). The other frequently reported $\mathrm{AE}$ was hypoglycemia ( $12.05 \%$ for vildagliptin, $10.3 \%$ for sitagliptin, and $7.29 \%$ for linagliptin). There were very low incidences of renal and hepatic toxicity, infections, and chest discomfort. There was no significant difference between groups in terms of reported AEs (Table 2).

\section{Discussion}

The inability of monotherapy to maintain good glycemic control in T2DM as a result of progressive deterioration of $\beta$-cell function provides the rationale for the early use of combination therapy with different classes of drugs. For the same reason, insulin therapy is frequently required to achieve sufficient glycemic control. However, insulin therapy may lead to weight gain, increasing risk of hypoglycemia, edema, and some other side effects [23]. Considering all these benefits and harms, the chosen therapeutic regimen must be balanced to achieve glycemic control and decrease the dose of insulin needed.

This 12-week, randomized, open-label, parallel study evaluated the efficacy and safety of vildagliptin, sitagliptin, or linagliptin in Chinese patients with T2DM inadequately controlled on dual combination of insulin and a traditional OHA. All the groups achieved a better glycemic control compared with baseline both at weeks 6 and 12. The FPG 


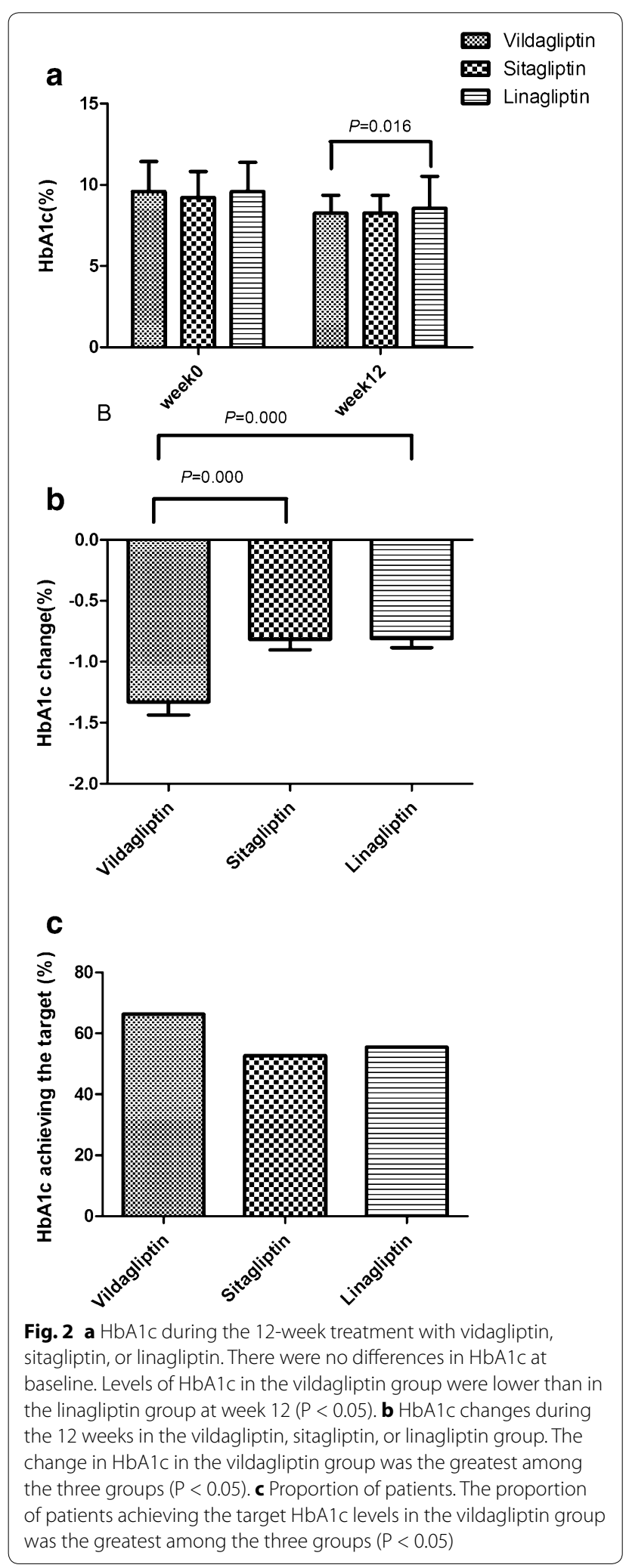

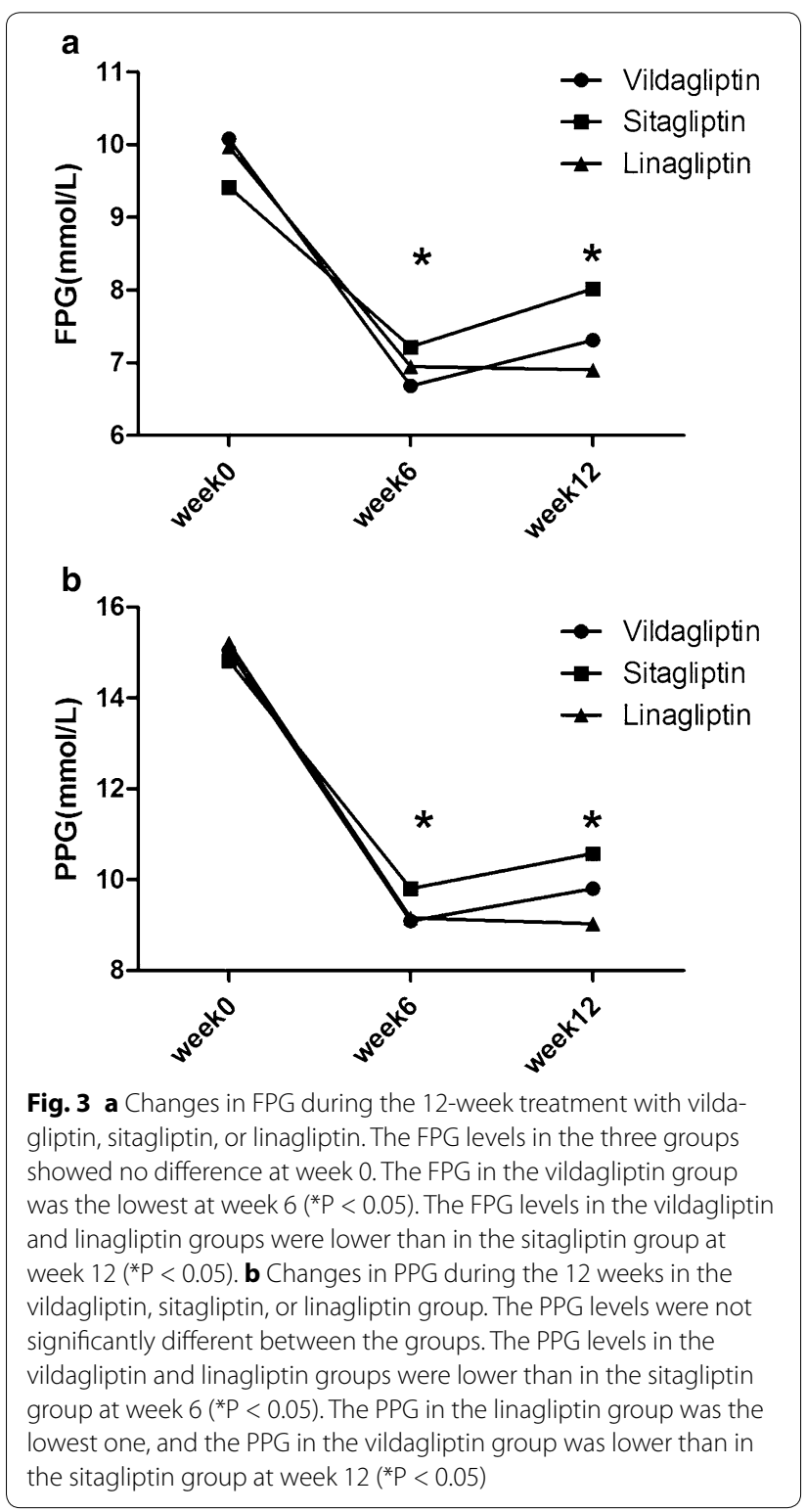

values decreased in the three groups after treatment with vildagliptin, sitagliptin, or linagliptin but to a slightly different degree. At week 12, vildagliptin and linagliptin induced a significantly greater decrease in FPG than sitagliptin did. Similarly, vildagliptin and linagliptin induced significantly greater decline in PPG when compared with sitagliptin. At week 12, patients treated with linagliptin revealed the most remarkable decrease in the PPG levels, followed by vildagliptin. The three DPP-4 inhibitors showed excellent effect on glycemic control as add-on therapy in treating T2DM. Our results are in accordance with previous 


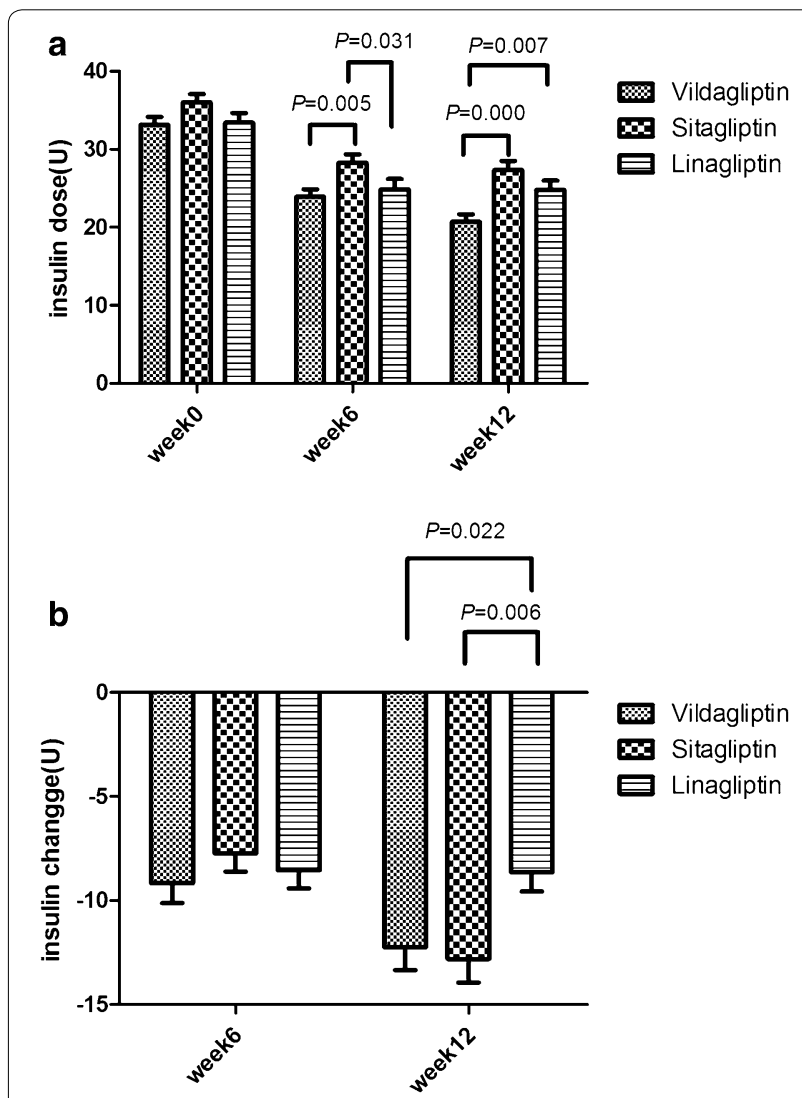

Fig. 4 a Insulin doses during the 12-week treatment with vildagliptin, sitagliptin, or linagliptin. The insulin doses at week 0 were not significantly different. The insulin dose in the vildagliptin group was lower than in the sitagliptin group at week 6 . The insulin dose in the linagliptin group was lower than in the sitagliptin group at week 6 .

The mean insulin dose in the vildagliptin group was much lower than in the other two groups at week $12(P<0.05)$. b Insulin changes during the 12-week treatment with vildagliptin, sitagliptin, or linagliptin. There were no significant differences between the three groups at week 6 . The insulin changes in the vildagliptin and sitagliptin groups were much more pronounced than in the linagliptin group $(P<0.05)$. FPG fasting plasma glucose, $H b A 1 c$ glycosylated hemoglobin, PPG postprandial plasma glucose

reports $[16,18,24]$. The present study showed vildagliptin, sitagliptin, and linagliptin help decrease FPG and PPG as add-on therapy to the background insulin treatment.
Our primary study end point was the change in HbA1c during the follow-up. At week 12 all three groups achieved significant change in HbA1c but the change in the vildagliptin arm was statistically greater than in the other two groups. Several previous reports demonstrated that vildagliptin, sitagliptin, and linagliptin are efficacious in decreasing HbA1c and enabled patients to reach glycemic control targets both as monotherapy and combination therapy [18, 19, 25-27]. Our study results are similar to those of previous studies in these regards. Moreover, as far as we know, this study is the first to compare vildagliptin, sitagliptin, and linagliptin as add-on therapy for T2DM patients with background insulin treatment. And what is more, our results indicate that vildagliptin is more effective in decreasing HbA1c.

It is generally acknowledged that high insulin doses might cause various unwanted effects during the treatment of T2DM. The present study extended the DPP-4 inhibitor treatment period to 12 weeks so that we were able to see the gradual decrease in blood glucose and the consequent decline in HbA1c. The gradual decline of blood glucose substantially decreased insulin requirement and resulted in considerable insulin dose reduction. Insulin doses in the vildagliptin group at weeks 6 and 12 were the lowest among the three groups. Additionally, the changes in insulin doses in the vildagliptin and sitagliptin groups were much more pronounced than in the linagliptin group.

The classical mechanism of the DPP-4 inhibitors to achieve glycemic control is based on the notion that these drugs increase the active levels of incretin hormones, GLP-1 and glucose-dependent insulinotropic polypeptide (GIP), and thereby improving pancreatic A- and B cell sensitivity to glucose [28]. A growing body of evidence both from clinical and preclinical findings supports the notion that DPP-4 inhibitors can ameliorate insulin resistance through several pathways $[29,30]$. Thus, these findings might be potential explanations as to why adding DPP-4 inhibitors to background insulin therapy could considerably decrease the required dosage of insulin.

The safety of DPP-4 inhibitors as add-on therapy to background insulin treatment in T2DM patients was one

Table 2 Adverse events during the 12 weeks

\begin{tabular}{|c|c|c|c|c|c|}
\hline Variables, n (\%) & Vildagliptin ( $n=166$ ) & Sitagliptin $(n=165)$ & Linagliptin $(n=164)$ & $\mathrm{x}^{2}$ values & $P$ values \\
\hline Hypoglycemia & $20(12.05)$ & $17(10.30)$ & $13(7.29)$ & 1.554 & 0.460 \\
\hline Gastrointestinal adverse events & $24(14.46)$ & $19(11.52)$ & $15(9.15)$ & 2.260 & 0.323 \\
\hline Renal and hepatic toxicity & $6(3.61)$ & $5(3.30)$ & $2(1.22)$ & 2.008 & 0.366 \\
\hline Infections & $10(6.02)$ & $8(4.85)$ & $12(7.32)$ & 0.881 & 0.644 \\
\hline Chest discomfort & $8(4.82)$ & $11(6.70)$ & $11(6.71)$ & 0.676 & 0.713 \\
\hline
\end{tabular}


of the relevant aspects being evaluated in the present study. The main AEs included gastrointestinal AEs, hypoglycemia, renal and hepatic toxicity, infections (including upper respiratory and urinary tract infections and nasopharyngitis), and chest discomfort. During this study, no pancreatitis was reported. The risk of hypoglycemia was similar in the three groups. As previously reported in meta-analyses, the rate of hypoglycemia was not higher in T2DM patients treated with the DPP-4 inhibitors, even in patients already undergoing treatment with insulin or a sulfonylurea [31, 32]. In accordance with our results, a meta-analysis reported that there was no increased incidence of gastrointestinal disorders in patients treated with DPP-4 inhibitors [33]. Moreover, the rates of other AEs in the present study were low and were not significantly different between the three groups.

The present study had certain limitations that need to be recognized. The study was performed in a relatively small number of patients at a single institution. Further studies are required to evaluate the long-term effects of DPP-4 inhibitors with or without insulin on glycemic control and insulin secretion in a larger number of patients. We did not recruit a group without DPP-4 inhibitors as controls, which is also a limitation to this study. Additionally, close pharmacovigilance monitoring plans are necessary to address the uncertainty regarding AEs of DPP-4 inhibitors, while their potential impact on cardiovascular outcomes will be clarified in the near future after the completion of more relevant long-term studies.

\section{Conclusions}

Vildagliptin, sitagliptin, or linagliptin appear to be effective and safe as add-on treatment for T2DM patients inadequately controlled on dual combination of insulin and another OHA. This is the first study to evaluate the efficacy and safety of this treatment regimen in Chinese T2DM patients. The three DPP-4 inhibitors had similar efficacy in achieving glycemic control, but vildagliptin was much more efficacious in decreasing insulin requirement and achieving target $\mathrm{HbA1c}$ level. Therefore, we consider that vildagliptin or any of the other tested DPP-4 inhibitors could be added to combination therapy to reach the glycemic control in inadequately controlled patients.

\footnotetext{
Abbreviations

ALT: alanine transaminase; AST: aspartate transaminase; BMI: body mass index; BUN: blood urea nitrogen; Cr: creatinine; DBP: diastolic blood pressure; FPG: fasting plasma glucose; GIP: glucose-dependent insulinotropic polypeptide; GLP-1: glucagon-like peptide-1; HbA1c : glycosylated hemoglobin; OHA: oral hypoglycemic agent; PPG: postprandial plasma glucose; SBP: systolic blood pressure; TC: total cholesterol; TG: triglyceride; ULN: upper limit of normal.
}

\section{Authors' contributions}

YZT conceived of the study, analyzed the data, drafted the manuscript.GW and $\mathrm{ZHJ}$ recruited the patients, collected the data, involved in the visiting of the patients. TTY, YJC, MY, LLM, YJZ, CGL, ZL, PY participated in the collection of data, involved in the visiting of the patients. CLN conceived of the study, and participated in its design and coordination and helped to draft the manuscript. All authors read and approved the final manuscript.

\section{Acknowledgements}

We would like to thank Professor Dai-Qing Li for valuable suggestions to the study and revising the manuscript. We would like to thank the nurses in the cardiovascular department of our hospital for collecting the blood samples.

\section{Competing interests}

We wish to confirm that there are no known conflicts of interest associated with this publication and there has been no significant financial support for this work that could have influenced its outcome. We confirm that the manuscript has been read and approved by all named authors and that there are no other persons who satisfied the criteria for authorship but are not listed. We further confirm that the order of authors listed in the manuscript has been approved by all of us. We confirm that we have given due consideration to the protection of intellectual property associated with this work and that there are no impediments to publication, including the timing of publication, with respect to intellectual property. In so doing we confirm that we have followed the regulations of our institutions concerning intellectual property. We further confirm that any aspect of the work covered in this manuscript that has involved human patients has been conducted with the ethical approval of all relevant bodies and that such approvals are acknowledged within the manuscript.

Received: 17 June 2015 Accepted: 8 October 2015

Published online: 19 October 2015

\section{References}

1. World Health Organization. Diabetes key facts. http://www.who.int/ mediacentre/factsheets/fs312/en/.

2. Go AS, Mozaffarian D, Roger VL, Benjamin EJ, Berry JD, Borden WB, Bravata DM, Dai S, Ford ES, Fox CS, et al. Heart disease and stroke statistics - 2013 update: a report from the American Heart Association. Circulation. 2013;127(1):e6-245

3. Yang W, Lu J, Weng J, Jia W, Ji L, Xiao J, Shan Z, Liu J, Tian H, Ji Q, et al. Prevalence of diabetes among men and women in China. N Engl J Med. 2010;362(12):1090-101.

4. Hayward RA, Reaven PD, Wiitala WL, Bahn GD, Reda DJ, Ge L, McCarren M, Duckworth WC, Emanuele NV. Follow-up of glycemic control and cardiovascular outcomes in type 2 diabetes. N Engl J Med. 2015;372(23):2197-206.

5. Leslie RD. United Kingdom prospective diabetes study (UKPDS): what now or so what? Diabetes/Metab Res Rev. 1999;15(1):65-71.

6. Feinkohl I, Keller M, Robertson CM, Morling JR, MCLachlan S, Frier BM, Deary IJ, Strachan MW, Price JF. Cardiovascular risk factors and cognitive decline in older people with type 2 diabetes. Diabetologia. 2015;58(7):1637-45.

7. Inzucchi SE, Bergenstal RM, Buse JB, Diamant M, Ferrannini E, Nauck M, Peters AL, Tsapas A, Wender R, Matthews DR. Management of hyperglycemia in type 2 diabetes: a patient-centered approach: position statement of the American Diabetes Association (ADA) and the European Association for the Study of Diabetes (EASD). Diabetes Care. 2012;35(6):1364-79.

8. Golightly LK, Drayna CC, McDermott MT. Comparative clinical pharmacokinetics of dipeptidyl peptidase-4 inhibitors. Clin Pharmacokinet. 2012;51(8):501-14.

9. Pratley RE, Nauck MA, Bailey T, Montanya E, Filetti S, Garber AJ, Thomsen $A B$, Furber S, Davies M. Efficacy and safety of switching from the DPP-4 inhibitor sitagliptin to the human GLP-1 analog liraglutide after 52 weeks 
in metformin-treated patients with type 2 diabetes: a randomized, openlabel trial. Diabetes Care. 2012;35(10):1986-93.

10. Zinman B. Initial combination therapy for type 2 diabetes mellitus: is it ready for prime time? Am J Med. 2011;124(1):S19-34.

11. Bailey T. Options for combination therapy in type 2 diabetes: comparison of the ADA/EASD position statement and AACE/ACE algorithm. Am J Med. 2013;126(9 Suppl 1):009.

12. Ji LN, Pan CY, Lu JM, Li H, Li Q, Li QF, Peng YD, Tian HM, Yao C, Zhao ZG, et al. Efficacy and safety of combination therapy with vildagliptin and metformin versus metformin up-titration in Chinese patients with type 2 diabetes mellitus: study design and rationale of the vision study. Cardiovasc Diabetol. 2013;12:118.

13. Odawara M, Hamada I, Suzuki M. Efficacy and safety of vildagliptin as add-on to metformin in Japanese patients with type 2 diabetes mellitus. Diabetes Ther Res Treat Educ Diabetes Relat Disord. 2014;5(1):169-81.

14. Ahren B, Foley JE, Bosi E. Clinical evidence and mechanistic basis for vildagliptin's action when added to metformin. Diabetes Obes Metab. 2011;13(3):193-203.

15. Godinho R, Mega C, Teixeira-de-Lemos E, Carvalho E, Teixeira F, Fernandes $R$, Reis F. The place of dipeptidyl peptidase-4 inhibitors in type 2 diabetes therapeutics: a "Me Too" or "the Special One" antidiabetic class? J Diabetes Res. 2015;2015:806979.

16. Kozlovski P, Foley J, Shao Q, Lukashevich V, Kothny W. Vildagliptin-insulin combination improves glycemic control in Asians with type 2 diabetes. World J Diabetes. 2013;4(4):151-6.

17. Murakami T, Nambu T, Kato T, Matsuda Y, Yonemitsu S, Muro S, Oki S. Addon treatment with mitiglinide improves residual postprandial hyperglycemia in type 2 diabetic patients receiving the combination therapy with insulin glargine and sitagliptin. Tohoku J Exp Med. 2015;235(4):255-60.

18. Katsuno T, Ikeda H, Ida K, Miyagawa J, Namba M. Add-on therapy with the DPP-4 inhibitor sitagliptin improves glycemic control in insulintreated Japanese patients with type 2 diabetes mellitus. Endocr J. 2013;60(6):733-42.

19. Aroda VR, Henry RR, Han J, Huang W, DeYoung MB, Darsow T, Hoogwerf BJ. Efficacy of GLP-1 receptor agonists and DPP-4 inhibitors: meta-analysis and systematic review. Clin Ther. 2012;34(6):1247-58.

20. Deacon CF, Mannucci E, Ahren B. Glycaemic efficacy of glucagon-like peptide-1 receptor agonists and dipeptidyl peptidase-4 inhibitors as add-on therapy to metformin in subjects with type 2 diabetes-a review and meta analysis. Diabetes Obes Metab. 2012;14(8):762-7.

21. Fakhoury WK, Lereun C, Wright D. A meta-analysis of placebo-controlled clinical trials assessing the efficacy and safety of incretin-based medications in patients with type 2 diabetes. Pharmacology. 2010;86(1):44-57.
22. Society CD. China guideline for type 2 diabetes (2013). Clin J Diabetes. 2014;22:865-8

23. UK Prospective Diabetes Study (UKPDS) Group. Intensive blood-glucose control with sulphonylureas or insulin compared with conventional treatment and risk of complications in patients with type 2 diabetes (UKPDS 33. Lancet. 1998;352(9131):837-53.

24. Gomis R, Espadero RM, Jones R, Woerle HJ, Dugi KA. Efficacy and safety of initial combination therapy with linagliptin and pioglitazone in patients with inadequately controlled type 2 diabetes: a randomized, double-blind, placebo-controlled study. Diabetes Obes Metab. 2011;13(7):653-61.

25. Guedes EP, Hohl A, de Melo TG, Lauand F. Linagliptin: farmacology, efficacy and safety in type 2 diabetes treatment. Diabetol Metab Syndr. 2013;5(1):25.

26. Haak T, Meinicke T, Jones R, Weber S, von Eynatten M, Woerle HJ. Initial combination of linagliptin and metformin in patients with type 2 diabetes: efficacy and safety in a randomised, double-blind 1-year extension study. Int J Clin Pract. 2013;67(12):1283-93.

27. Lipowsky C, Sze L, Krull I, Brandle M. Liraglutide as add-on therapy to insulin in type 2 diabetes mellitus: a retrospective, observational study from a daily clinical practice setting in Switzerland. Diabetes Ther Res Treat Educ Diabetes Related Disord. 2015;6(1):41-7.

28. Duttaroy A, Voelker F, Merriam K, Zhang X, Ren X, Subramanian K, Hughes TE, Burkey BF. The DPP-4 inhibitor vildagliptin increases pancreatic beta cell mass in neonatal rats. Eur J Pharmacol. 2011;650(2-3):703-7.

29. Aroor AR, Habibi J, Ford DA, Nistala R, Lastra G, Manrique C, Dunham MM, Ford KD, Thyfault JP, Parks EJ, et al. Dipeptidyl peptidase-4 inhibition ameliorates Western diet-induced hepatic steatosis and insulin resistance through hepatic lipid remodeling and modulation of hepatic mitochondrial function. Diabetes. 2015;64(6):1988-2001.

30. Kusunoki M, Sato D, Nakamura T, Oshida Y, Tsutsui H, Natsume Y, Tsutsumi K, Miyata T. DPP-4 inhibitor teneligliptin improves insulin resistance and serum lipid profile in japanese patients with type 2 diabetes. Drug Res. 2014;4:4.

31. Monami M, lacomelli I, Marchionni N, Mannucci E. Dipeptydil peptidase-4 inhibitors in type 2 diabetes: a meta-analysis of randomized clinical trials. Nutr Metab Cardiovasc Dis Nmcd. 2010;20(4):224-35.

32. Goossen K, Graber S. Longer term safety of dipeptidyl peptidase-4 inhibitors in patients with type 2 diabetes mellitus: systematic review and meta-analysis. Diabetes Obes Metab. 2012;14(12):1061-72.

33. Cai L, Cai Y, Lu ZJ, Zhang Y, Liu P. The efficacy and safety of vildagliptin in patients with type 2 diabetes: a meta-analysis of randomized clinical trials. J Clin Pharm Ther. 2012;37(4):386-98.

\section{Submit your next manuscript to BioMed Central and take full advantage of:}

- Convenient online submission

- Thorough peer review

- No space constraints or color figure charges

- Immediate publication on acceptance

- Inclusion in PubMed, CAS, Scopus and Google Scholar

- Research which is freely available for redistribution

Submit your manuscript at

www.biomedcentral.com/submit

C BioMed Central 